\title{
Image Restoration for Shift Invariant Blurs-A Survey
}

\author{
Mayana J. Shah \\ Department of EC \& C. Engineering \\ CKPCET College of Engineering, Surat, India
}

\author{
Upena D. Dalal \\ Department of Electronics Engineering \\ S.V.National Institute of Technology, Surat, India
}

\begin{abstract}
Even though Image Restoration being a conventional problem in image processing, is still a demanding area and has always attracted the interest of research community. Image restoration algorithms are important as it serves a wide range of real world applications such as astronomy, medical imaging and photo editing are just a few which demands a good quality image for further high level processing. Image restoration methods aim to reduce the degradations that have occurred while the digital image was being obtained. All natural images have gone through some sort of degradation when they are acquired, processed or displayed because of sensor noise, camera misfocus, blur caused by relative motion between object \& camera, atmospheric turbulence and others. The paper deal with restoration of images degraded by linear space-invariant blurs. Paper presents mathematical modeling of linear shift-invariant image formation process, possible sources of degradation, and reviews some fundamental \& specific methods of restoration.
\end{abstract}

\section{General Terms}

Algorithms, Modeling, Restoration.

\section{Keywords}

Blur, Spatial-Invariance, PSF, Deconvolution.

\section{INTRODUCTION}

Image restoration or deconvolution of a blurred natural image is a mature research activity with a rich set of available techniques and algorithms. Degradation of the image demands for the reconstruction of the original image [1]. Image restoration algorithms aim to recover the true image from degraded measurements. This inverse problem is typically illposed, meaning that the solution does not satisfy at least one of the following: existence, uniqueness, or stability. Regularization techniques are often adopted to obtain a solution with desired properties, indicating knowledge of prior information about the true image. Important performance metrics to assess the efficiency of restoration methods include: restoration accuracy, computational complexity and convergence speed. The paper provides overview of image restoration algorithms when degradation process is considered to be linear shift invariant.

The rest of the paper is arranged as follows. Image degradation model is discussed in Section 2. Various blur models are described in Section 3. Section 4 presents various fundamental \& specific (blind \& nonblind) scheme of image restoration. Conclusions are drawn in Section 5.

\section{IMAGE DEGRADATION MODEL}

In many imaging application ideal image is degraded version of original image as it is blurred by some function $\mathrm{h}$ which is called impulse response, blur or point spread function (PSF). PSF is the smallest image detail an imaging system can form. The wide range of degradations considered are atmospheric distortions, optical aberrations, sensor blur, motion blur resulting from camera shake or the movements of the objects in the scene and noise. Noises may be introduced by the transmission medium, the recording medium, measurement errors due to the limited accuracy of the recording system, quantization of the data for digital storage or any combination of these [3]. Degradation process is in general nonlinear and space varying but large number of problems could be addressed with a linear and shift-invariant (LSI) model considering every point in image equally blurred. Output of an LSI system is the convolution of the true image with the impulse response of the system as shown in Fig.1 (a).

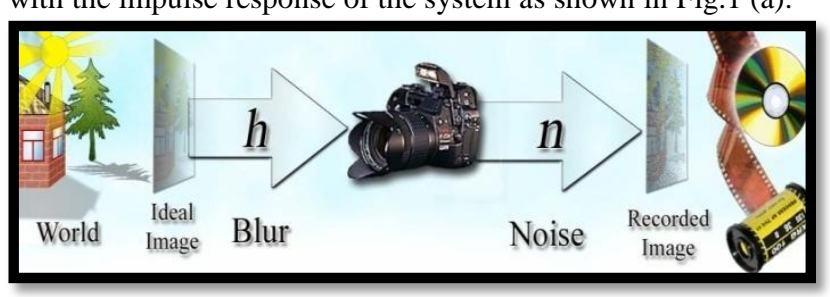

(a)

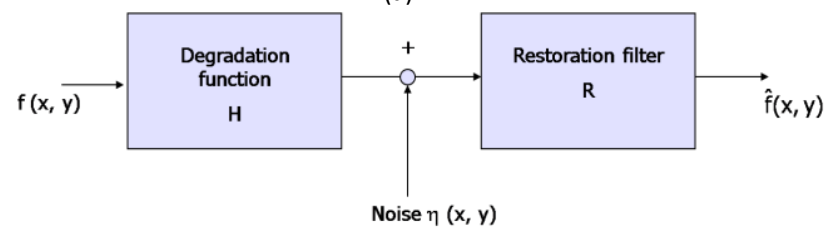

(b)

Fig.1: (a) Image Formation (b) Image degradation model. Suppose that $f(x, y)$ is the true image that we would like to recover from the degraded measurement $g(x, y)$, where $(x, y)$ indicates special coordinates. For a linear shift-invariant system, the imaging process can be formulated as

$$
g(x, y)=h(x, y) * f(x, y)+n(x, y)
$$

where "*" is the convolution operation, $\mathrm{h}(\mathrm{x}, \mathrm{y})$ is the PSF of the imaging system, and $\mathrm{n}(\mathrm{x}, \mathrm{y})$ is the additive noise. The block diagram of this process is given in fig. 1 (b).The imaging formulation can also be done in matrix-vector form or in frequency domain. Defining $\mathrm{g}, \mathrm{f}$, and $\mathrm{n}$ as vectorized versions of $g(x, y), f(x, y)$, and $n(x, y)$, respectively, the matrixvector formulation is given by:

$$
g=H f+n
$$

where $\mathrm{H}$ is a two-dimensional sparse matrix with elements taken from $\mathrm{h}(\mathrm{x}, \mathrm{y})$ to have the proper mapping from $\mathrm{f}$ to $\mathrm{g}$. On the other hand, the Fourier-domain version of the imaging model is given by:

$$
G(u, v)=H(u, v) F(u, v)+N(u, v)
$$

Where $G(u, v), H(u, v), F(u, v)$, and $N(u, v)$ are the Fourier transforms of $g(x, y), h(x, y), f(x, y)$, and $n(x, y)$ respectively [2].Various restoration methods optimized with respect to different criteria have been introduced in the paper.

\section{BLUR MODELS}

PSF can be represented by a convolution kernel. Every convolution kernel is a not valid PSF. Constraints PSF must satisfy are: i)The kernel h must satisfy the energy conservation constraint which states that the energy is neither lost nor gained by the blurring operation, ii)PSF is 
an energy distribution function so every convolution kernel that contains negative values is not a valid PSF.

\subsection{Out of focus Blur}

When a 3D scene is imagined onto a 2D imaging plane, some parts of the scene are in focus while other parts are not as shown in Fig.2.

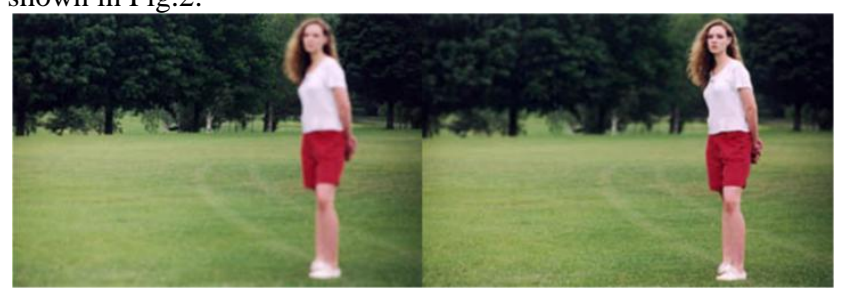

Fig.2: Illustration of out of focus Blur

If the aperture of the camera is circular, the image of any point source is a small disk, known as the circle of confusion (COC). The degree of defocus (diameter of the COC) depends on the focal length and the aperture number of the lens and the distance between camera and object. The spatially continuous PSF of this uniform out-of-focus blur with radius $\mathrm{R}$ is given by

$$
h(x, y)= \begin{cases}\frac{1}{\pi R^{2}}, & \text { if } \sqrt{x^{2}+y^{2}} \leq R^{2} \\ 0, & \text { otherwise }\end{cases}
$$

\subsection{Motion Blur}

It occurs when there is relative motion between the object and the camera during exposure. A camera works by exposing a sheet of light sensitive film to a scene for a short period of time. The light from the scene hits the film causing the film to change chemically which results in picture representation. If the scene changes during this exposure than blurring of image is occurred since light from many scene hit the film [4].

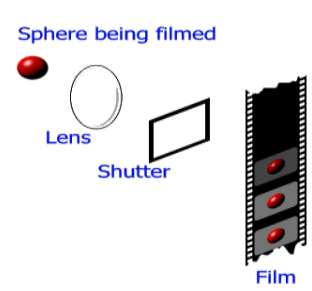

(a)

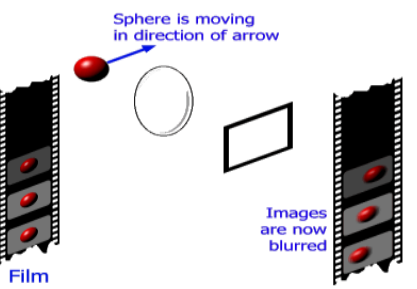

(b)
Fig.3: (a) Sphere being filmed (b) Sphere is moving in the direction of arrow.

If the $v_{\text {relative }}$ is the relative velocity between the camera \& the scene under an angle $\varphi$ radians with the horizontal axis during the exposure interval $\left[0, t_{\text {exposure }}\right]$. Defining the length of motion by $\mathrm{L}=\mathrm{v}_{\text {relative }} * \mathrm{t}_{\text {exposure }}$, than the PSF is given by;

$$
h(x, y, L, \phi)= \begin{cases}\frac{1}{L}, & \text { if }-\frac{L}{2} \leq i \leq \frac{L}{2}, x / y=-\tan ^{-1} \phi \\ 0, & \text { otherwise }\end{cases}
$$

Fig.3 (b) illustrates that the blur is effectively lowpass filtering operation.

\subsection{Atmospheric turbulence}

It is due to random variations in the reflective index of the medium between the object and the imaging system and it occurs in the imaging of astronomical objects.

$$
h(x, y)=K \exp \left(-\frac{x^{2}+y^{2}}{2 \sigma^{2}}\right)
$$

Here $\sigma$ determines the amount of spread of the blur, and the constant $\mathrm{K}$ is to be chosen so that constraint of PSF is satisfied.Fig. 4 illustrates atmospheric turbulence.

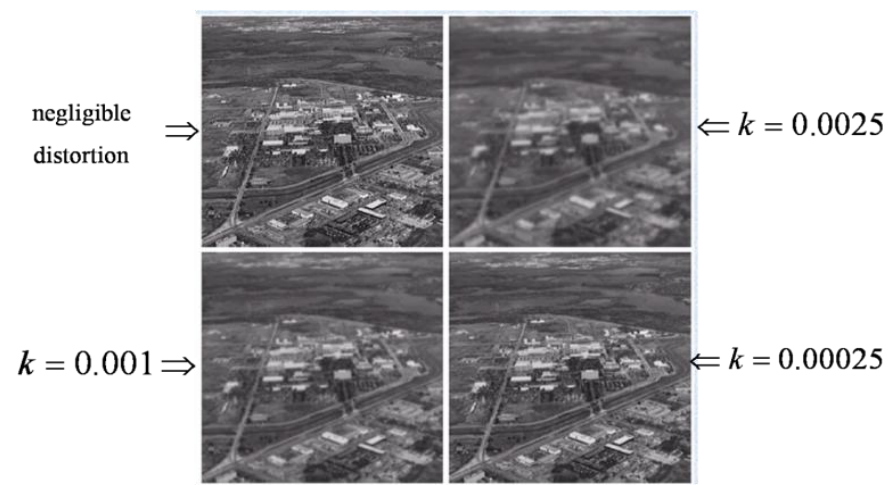

Fig. 4: Atmospheric turbulence for different values of $k$

\section{IMAGE RESTORATION METHODS}

Image Restoration techniques are broadly classified as nonblind technique if degradation process $\mathrm{H}$ is known or blind technique if degradation process $\mathrm{H}$ is unknown. Success of restoration algorithm depends on how accurately we model original image and the degradations.

\subsection{Inverse filtering}

If a good model of the blurring function $\mathrm{H}$ is known or can be developed, then inverse filtering is the quickest and easiest way to restore the blurred image. Since blurring is equivalent to low pass filtering of an image, inverse filtering provides with high pass filtering action to reconstruct the blurred image without much effort which require only the blur PSF as a priori knowledge. It perfectly restore the image when noise is absent. An inverse filter is a linear filter whose PSF $\operatorname{hinv}(\mathrm{n} 1, \mathrm{n} 2)$ is the inverse of the blurring function $\mathrm{h}(\mathrm{n} 1, \mathrm{n} 2)$ in the sense that

$$
\begin{aligned}
\operatorname{hinv} * \mathrm{~h} & =\sum_{\mathrm{k} 1=0}^{\mathrm{N}-1} \sum_{\mathrm{k} 2=0}^{\mathrm{M}-1} \operatorname{hinv}(\mathrm{k} 1, \mathrm{k} 2) \mathrm{h}(\mathrm{n} 1-\mathrm{k} 1, \mathrm{n} 2-\mathrm{k} 2) \\
& =\delta(\mathrm{n} 1, \mathrm{n} 2) \\
& \operatorname{Hinv}(\mathrm{u}, \mathrm{v}) \mathrm{H}(\mathrm{u}, \mathrm{v})=1 \Rightarrow \operatorname{Hinv}(\mathrm{u}, \mathrm{v})=1 / \mathrm{H}(\mathrm{u}, \mathrm{v})
\end{aligned}
$$

$M \& N$ represent image dimensions. Problem can be solved as a set of M X N scalar problems in Fourier domain as follows

$$
F(u, v)=\frac{H^{*}(u, v) G(u, v)}{|H(u, v)|^{2}} \Rightarrow f(i, j)=\mathfrak{J}^{-1}\left[\frac{H^{*}(u, v) G(u, v)}{|H(u, v)|^{2}}\right] \text { (9) }
$$

If $\mathrm{M}=\mathrm{N}$ and $\mathrm{H}^{-1}$ exists then in matrix notation

$$
\mathrm{f}=\mathrm{H}^{-1} \mathrm{~g}
$$

Computational issues concerning inverse filtering are: i) if the additive noise $N(u, v)$ is negligible than problem arises when $H(u, v)$ is very small, in that region inverse filtering can not be used. Solution to the problem is if these points are known they can be neglected in the computation of $F(u, v)$. ii) In the presence of external noise rewriting (9) as

$$
\hat{F}(u, v)=F(u, v)+\frac{N(u, v)}{H(u, v)}
$$

$N(u, v)$ dominates the result when $H(u, v)$ is small [6]. The solution is pseudoinverse filtering which carry out the restoration process in a limited neighbourhood about the origin where $H(u, v)$ is not very small. 


$$
\hat{F}(u, v)= \begin{cases}\frac{H^{*}(u, v) G(u, v)}{|H(u, v)|^{2}} & H(u, v) \geq T \\ 0 & H(u, v)<T\end{cases}
$$

where threshold $\mathrm{T}$ is defined by the user. To overcome noise sensitivity of the inverse filter a number of restoration filters have been developed that are called least square filters. The two most commonly used filters are Weiner filter \& constrained least square filter.

\subsection{Wiener filtering}

The Wiener filter is optimal in terms of the mean square error between the ideal and the restored image. Its objective is to minimize the following function,

$$
E\left\{(f-\hat{f})^{T}(f-\hat{f})\right\}
$$

To do so two conditions should hold, first one is,

$$
E\{\hat{\mathrm{f}}\}=E\{\mathrm{f}\} \Rightarrow E\{\mathrm{f}\}=\mathrm{W} E\{\mathrm{y}\}
$$

The second condition is indicates that error must be orthogonal to the observation about the mean

$$
E\left\{(\hat{f}-f)(y-E\{y\})^{T}\right\}=0
$$

In frequency domain solution is given by,

$$
H_{\text {wiener }}(\mathrm{u}, \mathrm{v})=\frac{S_{f f}(u, v) H^{*}(u, v)}{S_{f f}(u, v)|H(u, v)|^{2}+S_{n n}(u, v)}
$$

In frequency domain reconstructed image is given by;

$$
\hat{F}(u, v)=\frac{S_{f f}(u, v) H^{*}(u, v)}{S_{f f}(u, v)|H(u, v)|^{2}+S_{n n}(u, v)} G(u, v)
$$

Here $H^{*}(u, v)$ is the complex conjugate of $H(u, v)$, and $S_{f f}(u, v)$ $\& S_{n n}(u, v)$ are the power spectrum of the ideal image and the noise respectively. The power spectrum is a measure for the average signal power per spatial frequency $(u, v)$ carried by the image. For spatial frequencies where $S_{n n}(u, v)<<S_{f f}(u, v)$, the Wiener filter approaches to the inverse filter $\&$ it works as frequency rejection filter when $S_{n n}(u, v)>>S_{f f}(u, v)$. If the noise is white noise, its power spectrum $S_{n n}(u, v)$ is determined by the noise variance $\sigma_{\mathrm{n}}^{2}$ for all $(\mathrm{u}, \mathrm{v})$. The estimation of $S_{f f}(u, v)$ is somewhat more problematic since the ideal image is not available. The power spectrum of original image obtained in three ways: i) we can replace $S_{f f}(u, v)$ by an estimate of the power spectrum of the blurred image and compensate for the variance of the noise. ii) Estimate the power spectrum from a set of representative images iii) use a statistical model for the ideal image [5].

\subsection{Constrained Least Square Filter}

The least squares estimator minimizes the sum of squared differences between the real observation $\mathrm{g}(\mathrm{x}, \mathrm{y})$ and the predicted observation $h(x, y) * f(x, y)$. In spatial domain cost function to be minimized can be written as,

$$
\sum_{(\mathrm{x}, \mathrm{y})}|\mathrm{g}(\mathrm{x}, \mathrm{y})-\mathrm{h}(\mathrm{x}, \mathrm{y}) * \mathrm{f}(\mathrm{x}, \mathrm{y})|^{2}
$$

Its Fourier domain version,

In matrix-vector notation,

$$
\sum_{(u, v)}|G(u, v)-H(u, v) F(u, v)|^{2}
$$

Where $\mathrm{H}+$ is

$$
\hat{\mathrm{f}}=\arg \min _{\mathrm{f}}\|\mathrm{g}-\mathrm{Hf}\|^{2}=\mathrm{H}^{+} \mathrm{g}
$$

$$
\mathrm{H}^{+}=\left(\mathrm{H}^{\mathrm{T}} \mathrm{H}\right)^{-1} \mathrm{H}^{\mathrm{T}}
$$

\begin{tabular}{|c|c|c|}
\hline Type & Definition & Remarks \\
\hline Tihkhonov & $\begin{array}{c}\quad \phi(f)=\|\mathrm{Lf}\|^{2} \\
\mathrm{~L}=\text { Highpass operator }\end{array}$ & $\begin{array}{l}\text {-image } \\
\text { will be } \\
\text { blur }[7,8] .\end{array}$ \\
\hline $\begin{array}{c}\text { Total } \\
\text { Variation }\end{array}$ & $\begin{array}{l}\qquad \phi(f)=\|\nabla f\|_{1} \\
\text { Uses L1 norm of the } \\
\text { magnitude of gradient. }\end{array}$ & $\begin{array}{l}\text {-Edges are } \\
\text { preserved } \\
{[9]}\end{array}$ \\
\hline $\begin{array}{c}\text { Bilateral Total } \\
\text { Variation }\end{array}$ & $\begin{array}{c}\phi(\underline{f})=\underbrace{f}_{\sum_{l=-P}^{\sum_{m=0}^{P}} \sum^{P} \alpha^{|m|+|l|} \| f-s_{x}^{l} s_{y}^{m}} \|_{1} \\
s_{x}^{l}, s_{y}^{m}=\text { shift } \mathrm{f} \text { by } 1 \text { and } \mathrm{m} \\
\text { pixels in horizontal and } \\
\text { vertical directions } \\
\text { respectively. }\end{array}$ & $\begin{array}{l}\text {-It is fast. } \\
\text {-Edges are } \\
\text { preserved } \\
{[10] .}\end{array}$ \\
\hline $\begin{array}{l}\text { Dictionary } \\
\text { learning }\end{array}$ & $\begin{array}{l}\phi \phi(f)=1^{\mathrm{T}} \rho(\Phi f) \\
\text { The } \Phi \text { matrix could represent } \\
\text { operations such as gradient, } \\
\text { Laplacian, discrete cosine } \\
\text { transform (DCT), and wavelet } \\
\text { decomposition }\end{array}$ & $\begin{array}{c}\text { - good } \\
\text { restoration } \\
\text { as we can } \\
\text { choose or } \\
\text { learn the } \\
\text { dictionary } \\
\text { [11] }\end{array}$ \\
\hline $\begin{array}{c}\text { Entropy } \\
\text { maximization }\end{array}$ & $\begin{array}{c}\phi(\mathrm{f})=\sum_{\mathrm{x}, \mathrm{y}} \mathrm{f}(\mathrm{x}, \mathrm{y}) \log (\mathrm{f}(\mathrm{x}, \mathrm{y})) \\
\text { Entropy function can be the } \\
\text { intensities or the gradients. }\end{array}$ & $\begin{array}{l}\text { - requires } \\
\text { iterative } \\
\text { methods. } \\
\text { [12] }\end{array}$ \\
\hline $\begin{array}{c}\text { Markov } \\
\text { random field }\end{array}$ & $\begin{array}{c}\phi(f)=\sum_{c \in C} V_{c}(f) \\
\text { potential function } V_{c}(f) \\
\text { defines local interactions of } \\
\text { clique c \&C is set of all } \\
\text { cliques }\end{array}$ & $\begin{array}{l}\text { - structural } \\
\text { properties } \\
\text { can be } \\
\text { modeled } \\
\text { based on } \\
\mathrm{V}_{\mathrm{c}}(\mathrm{f}) \\
\end{array}$ \\
\hline
\end{tabular}

The constraint $\mathrm{g}=\mathrm{Hf}$ is considered too strict because of noise and inaccuracies in the PSF estimate, and so the constraint is relaxed. In general, the regularized least squares estimation is given by:

$$
\hat{\mathrm{f}}=\arg \min _{\mathrm{f}}\left\{\|\mathrm{g}-\mathrm{Hf}\|^{2}+\lambda \phi(\mathrm{f})\right\}
$$

Various regularization parameters are tabulated in Table I.

Table I. Various regularization parameters

Constrained least squares (CLS) restoration with Tikhonov regularization can be formulated by choosing an $\mathrm{f}$ to minimize the Lagrangian

$$
L(f)=\|g-H f\|^{2}+\lambda\|L f\|^{2}
$$

Where $L(f)$ is a high pass filtered version of the image which imposes smoothness on the solution. This constraint controls the highpass version of the image which contains a considerably large amount of noise \& can be handled using optimization techniques. The original image estimate after minimization of the function becomes;

$$
\hat{\mathrm{f}}=\left(\mathrm{H}^{\mathrm{T}} \mathrm{H}+\lambda \mathrm{L}^{\mathrm{T}} \mathrm{L}\right)^{-1} \mathrm{H}^{\mathrm{T}} \mathrm{g}
$$

$\lambda$ is a positive regularization parameter used to control the trade-off between the fidelity and regularization terms. With 
larger values of $\lambda$, we get more regularisation, the restored image tends to have more ringing present near edges in the restored image [13]. With smaller values of $\lambda$, the restored image tends to have more amplified noise effects. In fourier domain;

$$
\hat{\mathrm{F}}(\mathrm{u}, \mathrm{v})=\frac{\mathrm{H}^{*}(\mathrm{u}, \mathrm{v}) \mathrm{G}(\mathrm{u}, \mathrm{v})}{|\mathrm{H}(\mathrm{u}, \mathrm{v})|^{2}+\lambda|\mathrm{L}(\mathrm{u}, \mathrm{v})|^{2}}
$$

\subsection{Steepest Descent Iterative Method}

An iterative scheme is used when there is no direct solution or a direct solution is not computationally feasible. For iterative scheme new estimate is given by old estimate plus some function of old estimate. The steepest descent method updates an initial estimate iteratively in the reverse direction of the gradient of the cost function C(f ) [14]. An iteration of the steepest descent method is given by;

$$
\mathrm{f}^{(\mathrm{i}+1)}=\mathrm{f}^{(\mathrm{i})}-\left.\alpha \frac{\partial \mathrm{C}(\mathrm{f})}{\partial \mathrm{f}}\right|_{\mathrm{f}^{(\mathrm{i})}}
$$

Where $\alpha$ is the step size and $\mathrm{f}^{(\mathrm{i})}$ is the ith estimate. The cost function if regularization is not used is defined as;

$$
\mathrm{C}(\mathrm{f})=(1 / 2)\|\mathrm{g}-\mathrm{Hf}\|^{2}
$$

which result in to the iteration;

$$
\mathrm{f}^{(\mathrm{i}+1)}=\mathrm{f}^{(\mathrm{i})}-\left.\alpha \frac{\partial \mathrm{C}(\mathrm{f})}{\partial \mathrm{f}}\right|_{\mathrm{f}^{(\mathrm{i})}}=\mathrm{f}^{(\mathrm{i})}+\alpha \mathrm{H}^{\mathrm{T}}\left(\mathrm{g}-\mathrm{Hf}^{(\mathrm{i})}\right)
$$

which is also known as the Landweber iteration [15].The iterations are repeated until a convergence criterion is reached which is; i) the maximum number of iterations; ii) the rate of change in the estimated signal $\left\|\left(\mathrm{f}^{(\mathrm{i}+1)}\right)-\left(\mathrm{f}^{(\mathrm{i})}\right)\right\| /\left\|\mathrm{f}^{(\mathrm{i})}\right\|$ or in cost $\left|\mathrm{C}\left(\mathrm{f}^{(\mathrm{i}+1)}\right)-\mathrm{C}\left(\mathrm{f}^{(\mathrm{i})}\right)\right| / \mathrm{C}\left(\mathrm{f}^{(\mathrm{i})}\right)$ between two successive iterations. The step size $\alpha$ should be small enough to guarantee convergence, on the other hand, the convergence rate would be slow if it is too small [16]. To guarantee the convergence $\left|1-\alpha s_{\max }\right|<1 \Rightarrow 0<\alpha<2 / s_{\max }$, where $\mathrm{S}_{\max }$ is the maximum singular value of $\mathrm{H}^{\mathrm{T}} \mathrm{H}$. The conjugate gradient method is used to have faster convergence. In the steepest descent method the estimate is updated in the direction of the residual where as in the conjugate gradient method the update direction is conjugate to previous directions.

Advantage of iterative algorithms are: i) there is no need to explicitly implement the inverse of an operator, ii) the restoration process is monitored as it progresses, iii) termination of the algorithm may take place before convergence $\&$ the effects of noise can be controlled in each iteration, iv) the algorithms used can be spatially adaptive.

\subsection{Bayesian Approach}

Statistical perspective to the image restoration problem is provided by Bayesian approach where unknown image, noise, and PSF are viewed as random variables. The solution of the problem is obtained by maximum likelihood (ML) estimator or maximum a posteriori (MAP) estimator or minimum mean squared error (MMSE) estimator. ML solution maximizes the probability $\mathrm{p}(\mathrm{g} \mid \mathrm{f})$ while the maximum a posteriori (MAP) estimator maximizes $\mathrm{p}(\mathrm{f} \mid \mathrm{g})$. Using the Bayes rule, the MAP estimator can be written in terms of the conditional probability $\mathrm{p}(\mathrm{g} \mid \mathrm{f})$ and the prior probability of $\mathrm{p}(\mathrm{f})$;

$$
\begin{aligned}
\hat{f}=\arg \max _{\mathrm{f}} p(f \mid g) & =\arg \max _{\mathrm{f}} \frac{p(g \mid f) p(f)}{p(g)} \\
& =\arg \max _{\mathrm{f}} p(g \mid f) p(f)
\end{aligned}
$$

where $\mathrm{p}(\mathrm{g})$ is dropped from the last term as it is constant with respect to the argument $f$.The last term reveals that the MAP solution reduces to the ML solution when $p(f)$ is a uniform distribution. The minimum mean squared error (MMSE) estimator aims to find the expected value of $\mathrm{f}$, defined as;

$$
E[(f \mid g)]=\int f p(f \mid g) d f
$$

In image restoration problems image noise is mostly modelled to be a zero-mean independent identically distributed (iid) Gaussian random variable so the conditional probability of the observed image is;

$$
\begin{gathered}
\mathrm{p}(\mathrm{g} \mid \mathrm{f})=\frac{1}{\left(\sqrt{2 \pi} \sigma_{\mathrm{n}}\right)^{\mathrm{M}}} \exp \left(-\frac{1}{2 \sigma_{\mathrm{n}}^{2}} \sum_{\mathrm{x}, \mathrm{y}}(\mathrm{g}(\mathrm{x}, \mathrm{y})-\mathrm{h}(\mathrm{x}, \mathrm{y}) * \mathrm{f}(\mathrm{x}, \mathrm{y}))^{2}\right) \\
=\frac{1}{\left(\sqrt{2 \pi} \sigma_{\mathrm{n}}\right)^{\mathrm{M}}} \exp \left(-\frac{1}{2 \sigma_{\mathrm{n}}^{2}}\|\mathrm{~g}-\mathrm{Hf}\|_{2}^{2}\right)
\end{gathered}
$$

Where $\sigma_{\mathrm{n}}$ the noise standard deviation and $\mathrm{M}$ is is the total number of pixels in the observed in image. Well known iterative Richardson-Lucy algorithm is based on Poisson noise model $[17,18]$. Conditional probability of the observed image $\&$ an iteration of method are given by following equations;

$$
\begin{aligned}
& \mathrm{p}(\mathrm{g} \mid \mathrm{f})=\prod_{\mathrm{x}, \mathrm{y}} \frac{(\mathrm{h}(\mathrm{x}, \mathrm{y}) * \mathrm{f}(\mathrm{x}, \mathrm{y}))^{\mathrm{g}(\mathrm{x}, \mathrm{y})} \exp (-(\mathrm{h}(\mathrm{x}, \mathrm{y}) * \mathrm{f}(\mathrm{x}, \mathrm{y})))}{\mathrm{g}(\mathrm{x}, \mathrm{y}) !} \\
& \mathrm{f}^{(\mathrm{i}+1)}(\mathrm{x}, \mathrm{y})=\left[\frac{\mathrm{g}(\mathrm{x}, \mathrm{y})}{\mathrm{h}(\mathrm{x}, \mathrm{y}) * \mathrm{f}^{(\mathrm{i})}(\mathrm{x}, \mathrm{y})} * \mathrm{~h}^{\mathrm{T}}(\mathrm{x}, \mathrm{y})\right] \mathrm{f}^{(\mathrm{i})}(\mathrm{x}, \mathrm{y})
\end{aligned}
$$

Moacir P.et. al.have presented a restoration approach through band extrapolation [37]. An extrapolation algorithm using constraints on both spatial and frequency domains with a smoothing operator were combined with the Richardson-Lucy iterative algorithm.

\subsection{Projection onto Convex Set}

The projection onto convex sets (POCS) technique consists of iterative projection of an initial estimate onto predefined constraint sets [19]. Various popular constraint set used are; i) Data fidelity ii) pixel range constraint iii) frequency domain constraint set.

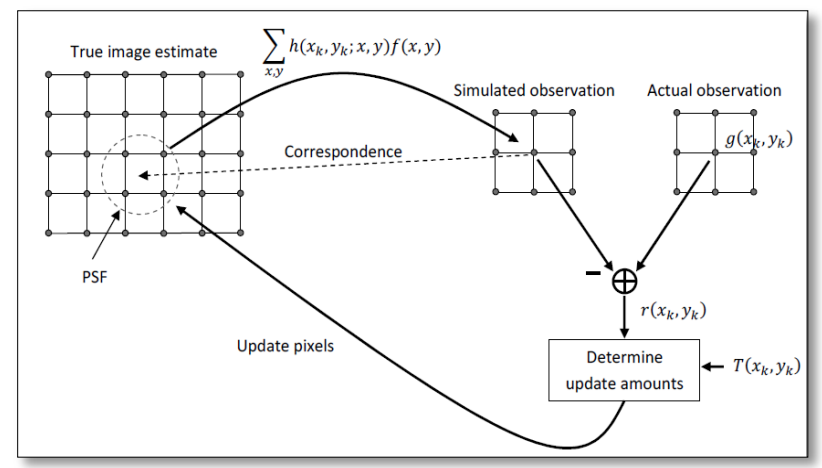

Fig.5: An illustration of the projection onto data fidelity constraint set

Data fidelity is commonly used as a constraint set to ensure consistency with observations and constrains the difference between the predicted pixel value and the observed pixel value.

$$
\mathrm{C}_{\mathrm{D}}=\left\{\mathrm{f}: \mid \mathrm{r}\left(\mathrm{x}_{\left.\mathrm{k}, \mathrm{y}_{\mathrm{k}}\right)}\right) \leq \mathrm{T}\left(\mathrm{x}_{\left.\left.\mathrm{k}, \mathrm{y}_{\mathrm{k}}\right)\right\}}\right.\right.
$$

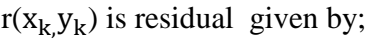

$$
r\left(x_{k}, y_{k}\right)=g\left(x_{k,} y_{k}\right)-\sum_{x, y \in S_{k}} h_{k}\left(x_{k}, y_{k} ; x, y\right) f(x, y)
$$

$S_{k}$ is the set of pixels in the $f(x, y)$ that contributes to the pixel $\left(x_{k}, y_{k}\right), h\left(x_{k}, y_{k} ; x, y\right)$ is the contribution of the pixel $f(x, y)$, 
and $T\left(x_{k}, y_{k}\right)$ is the threshold value that controls the data fidelity constraint. Selection of threshold should be proportional to the noise power [20]. According to data fidelity constraint current estimate is changed when the absolute value of the residual is less than the threshold $T\left(x_{k}\right.$, $y_{k}$ ) else it is updated. Fig.5 shows projection operation onto the data fidelity constraint set is formulated as; $\mathrm{f}^{(\mathrm{i}+1)}(\mathrm{x}, \mathrm{y})$

$$
=\left\{\begin{array}{c}
f^{(i)}(x, y)+\frac{\left(r\left(x_{k}, y_{k}\right)-T\left(x_{k}, y_{k}\right)\right) h\left(x_{k}, y_{k} ; x, y\right)}{\sum_{x, y \in S_{k}} h^{2}\left(x_{k} y_{k} ; x, y\right)}, r\left(x_{k}, y_{k}\right)>T\left(x_{k}, y_{k}\right) \\
f^{(i)}(x, y),\left|r\left(x_{k}, y_{k}\right)\right| \leq T\left(x_{k}, y_{k}\right) \\
f^{(i)}(x, y)+\frac{\left(r\left(x_{k}, y_{k}\right)+T\left(x_{k}, y_{k}\right)\right) h\left(x_{k}, y_{k} ; x, y\right)}{\sum_{x, y \in S_{k}} h^{2}\left(x_{k}, y_{k} ; x, y\right)}, r\left(x_{k}, y_{k}\right)<-T\left(x_{k}, y_{k}\right)
\end{array}\right.
$$

The pixel range constraint set ensures resulting image pixel values within a certain range. Frequency domain constraint sets is used when a certain frequency range of the true signal is known. Advantage of the POCS technique is the ease of incorporating space-variant PSF into the restoration. The technique guarantees convergence to a solution that is consistent with all the constraint sets when the constraint sets are convex and not disjoint but the solution is not necessarily unique $\&$ depend on the initial estimate and the order of the projections.

\subsection{Learning-Based Approach}

It uses powerful class-specific priors than generic image priors [21, 22]. It is used for both high-level classes and lowlevel classes. Constraining Solution to a Subspace is a one of training-based approach. If the image to be restored belongs to a class and there is a set of training images $\mathrm{f}_{1}, \mathrm{f}_{2} . . \mathrm{f}_{\mathrm{N}}$ from that class than image $\mathrm{f}$ can be represented in terms of the average image a and $\mathrm{K}$ basis vectors $\Psi=\left[\Psi_{1}, \Psi 2, \Psi_{\mathrm{K}}\right]$ :

$$
\hat{\mathrm{f}}=\mathrm{a}+\Psi \mathrm{e}
$$

here e shows the contributions of the basis vectors. Vector $\mathrm{e}$ size is smaller than the total pixels in $\mathrm{f}$. Vector e is calculated by taking the inner product with the basis vectors:

$$
\mathrm{e}=\Psi^{\mathrm{T}}(\mathrm{f}-\mathrm{a})
$$

Basis vectors are selected such that the difference between the image $f$ and its subspace representation $\hat{f}$ is made as small as possible. The restoration problem can be formulated in terms of the representation vector e. Optimal representation vector for the regularized least squares approach is given by:

$$
\hat{\mathrm{e}}=\arg \min _{\mathrm{e}}\|\mathrm{g}-\mathrm{H}(\mathrm{a}+\Psi \mathrm{e})\|^{2}+\lambda \phi(\mathrm{e})
$$

In this approach noise that is orthogonal to the subspace is automatically eliminated.

\subsection{Alternating Minimization}

In most of real life situation both the PSF and the true image must be estimated $\&$ this estimation process could be separate or joint. Estimation process uses various constraints such as nonnegativity, symmetry, and smoothness about the PSF and the true image. To find both the PSF $h$ and the true image $f$ that maximize the following posterior probability

$$
\mathrm{p}(\mathrm{f}, \mathrm{h} \mid \mathrm{g})=\frac{\mathrm{p}(\mathrm{g} \mid \mathrm{f}, \mathrm{h}) \mathrm{p}(\mathrm{f}) \mathrm{p}(\mathrm{h})}{\mathrm{p}(\mathrm{g})}
$$

wherep $(g \mid f, h)$ is the likelihood of observations, $p(f)$ is the image prior, and $\mathrm{p}(\mathrm{h})$ is the PSF prior. In optimization $\mathrm{p}(\mathrm{g})$ probability is dropped as it is constant with respect to $\mathrm{f} \&$ h. If parameterization is used for the prior distributions and introduced parameters are considered as random variables called hyperparameters than the posterior probability becomes:

$$
\mathrm{p}(\mathrm{f}, \mathrm{h}, \Omega \mid \mathrm{g})=\frac{\mathrm{p}(\mathrm{g} \mid \mathrm{f} . \mathrm{h}, \Omega) \mathrm{p}(\mathrm{f} \mid \Omega) \mathrm{p}(\mathrm{h} \mid \Omega) \mathrm{p}(\Omega)}{\mathrm{p}(\mathrm{g})}
$$

unknown image could be obtained as;

$$
\hat{\mathrm{f}}=\arg \max _{\mathrm{f}} \int_{\mathrm{h}, \Omega} \mathrm{p}(\mathrm{f}, \mathrm{h}, \Omega \mid \mathrm{g}) \mathrm{dhd} \Omega
$$

Instead of determining the optimal $\mathrm{f}$ and $\mathrm{h}$ simultaneously alternating minimization is used which alternatively updates the one variable while keeping the other fixed [23, 24]. Equations for next estimate of $\mathrm{f} \& \mathrm{~h}$ are as follows;

$$
\begin{aligned}
\mathrm{f}^{(\mathrm{i}+1)} & =\arg \min _{\mathrm{f}^{(\mathrm{i})}} \mathrm{C}\left(\mathrm{f}^{(\mathrm{i})}, \mathrm{h}^{(\mathrm{i})}\right) \\
\mathrm{h}^{(\mathrm{i}+1)} & =\arg \min _{\mathrm{h}^{(\mathrm{i})}} \mathrm{C}\left(\mathrm{f}^{(\mathrm{i}+1)}, \mathrm{h}^{(\mathrm{i})}\right)
\end{aligned}
$$

and the corresponding alternating minimization iterations are

$$
\begin{aligned}
& \mathrm{F}^{(\mathrm{i}+1)}(\mathrm{u}, \mathrm{v})=\frac{\mathrm{H}^{(\mathrm{i}) *}(\mathrm{u}, \mathrm{v}) \mathrm{G}(\mathrm{u}, \mathrm{v})}{\left|\mathrm{H}^{(\mathrm{i})}(\mathrm{u}, \mathrm{v})\right|^{2}+\lambda_{1}\left|\mathrm{~L}_{1}(\mathrm{u}, \mathrm{v})\right|^{2}} \\
& \mathrm{H}^{(\mathrm{i}+1)}(\mathrm{u}, \mathrm{v})=\frac{\mathrm{F}^{(\mathrm{i}+1) *}(\mathrm{u}, \mathrm{v}) \mathrm{G}(\mathrm{u}, \mathrm{v})}{\left|\mathrm{F}^{(\mathrm{i}+1)}(\mathrm{u}, \mathrm{v})\right|^{2}+\lambda_{2}\left|\mathrm{~L}_{2}(\mathrm{u}, \mathrm{v})\right|^{2}}
\end{aligned}
$$

Initial true image estimation obtained by applying a shock filter [25] or a bilateral filter [26] results in highly successful blind deconvolution algorithms with sharp edges in image.

\subsection{Iterative Blind Deconvolution}

Iterative blind deconvolution (IBD) is one of popular algorithm for blind deconvolution proposed by Dainty [27]. The IBD algorithm iteratively estimates the original image as well as the PSF as shown in Fig 6. IBD makes use of spatial domain (positivity) as well as frequency domain constraints $(G(u, v)=F(u, v) X H(u, v))$. In Fourier domain, the current estimate $\mathrm{F}^{(\mathrm{i})}$ is updated as;

$$
\mathrm{F}^{(\mathrm{i}+1)}(\mathrm{u}, \mathrm{v})=(1-\gamma) \mathrm{F}^{(\mathrm{i})}(\mathrm{u}, \mathrm{v})+\gamma \mathrm{G}(\mathrm{u}, \mathrm{v}) / \mathrm{H}^{(\mathrm{i})}(\mathrm{u}, \mathrm{v})
$$
which is essentially a weighted average of $F^{(i)}(u, v)$ and $\mathrm{G}(\mathrm{u}, \mathrm{v}) / \mathrm{H}^{(\mathrm{i})}(\mathrm{u}, \mathrm{v})$ with weight parameter $\gamma$.

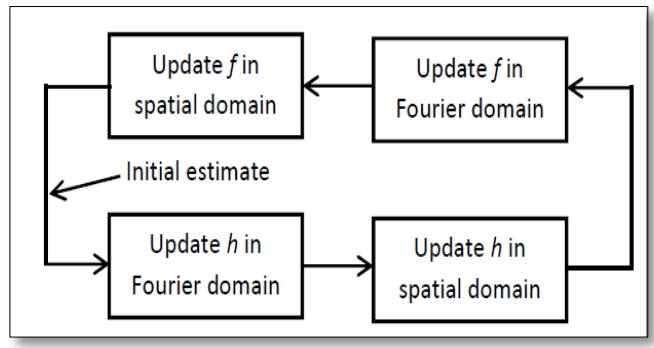

Fig. 6: IBD with spatial and Fourier domain constraints The iterative loop is repeated until a satisfactory restored image is obtained. Algorithm is sensitive to the initial guess \& may run into infinite loop without convergence [28, 29].

\subsection{Iterative Reweighted Least Squares (IRLS) Method}

The IRLS method [30] is designed to minimize $\ell p$ norm with $0<p \leq 1$ rewritten as weighted $\ell 2$ norm as $\left\|\mathrm{D}^{(\mathrm{i})+} \mathrm{f}^{(\mathrm{i})}\right\|_{2}^{2}$ where $\left|f^{(i)}\right|^{1-p / 2}$ is diagonal of matrix $D^{(i)+}$ with the current estimate is $f(i) \& D^{(i)+}$ as the pseudo-inverse of $D^{(i)}$. The $\ell p$ norm minimization problem becomes;

$$
\mathrm{f}^{(\mathrm{i}+1)}=(1 / 2)\|\mathrm{g}-\mathrm{Hf}\|^{2}+\lambda 1^{\mathrm{T}} \rho(\mathrm{f})
$$

The idea has been used in total variation-based restoration [31], where the regularizer is $\left\|D^{(i)+} \nabla f^{(i)}\right\|_{2}^{2}$. 


\subsection{Iterative Shrinkage Method}

Traditional optimization algorithms are not efficient when the regularizer is nonquadratic. IST algorithms have been recently developed to do $\ell 1$ minimization. In Proximal function IST technique cost function includes a distance term promoting the proximity between subsequent estimates [32]. The distance term is quadratic and convex, and leads to a simple and effective algorithm. The modified cost function is;

$\left.C(f)=0.5\|g-H f\|^{2}+\lambda 1^{T} \rho(f)+\mu / 2\right)\left\|f-f^{(i)}\right\|^{2}-(1 / 2)\left\|H f-H f^{(i)}\right\|^{2}(48)$ Where $\mu$ is chosen such that the distance term is strictly convex. Amir Beck introduced new fast iterative shrinkage thresholding algorithm with a global rate of convergence which is proven to be significantly better [38].

\subsection{Other Methods}

PDE Approach: Solutions to the image restoration uses theories on partial differential equations (PDEs) to develop new models representing an image $f(x, y, t)$ as a function of space and time, and starting with the original image at $\mathrm{t}=0$. the evolution of the image in time can be written by;

$$
\frac{\partial f(x, y, t)}{\partial t}+F\left(x, y, f(x, y, t), \nabla f(x, y, t), \nabla^{2} f(x, y, t)\right)
$$

where the functional $F$ takes spatial position, the image, and its first and second order spatial derivatives as the input. With the choice of the functional, it is possible to impose smoothness or sharpness on the restored image [33].

Total Least Squares: TLS approach is an extension of the least squares method which only allows perturbations in the observation g. The Total Least Squares (TLS) approach allows errors in both the measured (or estimated) PSF and the observation. The TLS problem is formulated as finding $\mathrm{f}, \delta \mathrm{g}$ and $\delta \mathrm{H}$ minimizing the Frobenius norm $\|\delta \mathrm{H}, \delta \mathrm{g}\| \mathrm{F}$ subject to the imaging constraint $\mathrm{g}+\delta \mathrm{g}=(\mathrm{H}+\delta \mathrm{H}) \mathrm{f}$, where $\delta \mathrm{H}$ and $\delta \mathrm{g}$ are the error components in the PSF and observation [34]. ARMA Parametric Approach: Auto regressive moving average (ARMA) approach is ARMA parameter estimation problem used for blind deconvolution which has been studied extensively [35]. ARMA model the true image as a twodimensional autoregressive (AR) process and the PSF as a two-dimensional moving average (MA) process. Critical issue with this approach is that global AR parameters may not accurately represent the entire image. W. Dong introduced adaptive regularization terms into the sparse representation framework. Autoregressive (AR) models are learned from the dataset of example image patches to find best fitted AR models to a given patch are adaptively selected to regularize the image local structures than the image non-local selfsimilarity is introduced as another adaptive regularization term [36].

\section{CONCLUSION}

The paper deal with restoration of images degraded by spaceInvariant blurs. Important and interesting image restoration techniques having potential for further development are discussed in brief. Various nonblind, blind (stastical, iterative, direct) methods discussed here provides foundation for image restoration which is going to maintain its importance as new devices, applications, and technologies continue to emerge and bring new challenges.

\section{REFERENCES}

[1] Banham, M. R. and Katsaggelos, A. K, “ Digital image restoration ", IEEE signal processing magazine, pp 2441, 1997.

[2] Bahadir Kursat Gunturk., and Xin Li, "Image Restoration: Fundamentals and Advances." CRC press, September 11, 2012

[3] Jiang, X., Cheng, D. C., Wachenfeld, S., Rothaus, K., “ Motion Deblurring", University of Muenster, Department of Mathematics and Computer Science, 2005

[4] Dr. Tania Stathaki, "Image Restoration", Imperial College of Science Technology and Medicine, Department of Electrical and Electronic Engineering, 2012

[5] Alan C. Bovik, "The Essential Guide to Image Processing."Academic Press, pp 324-348, July, 2009

[6] Gonzalez, R., Woods, R., and Eddins, S., "Digital Image Processing Using MATLAB." Pearson Prentice-Hall, Upper Saddle River, NJ, 2004.

[7] H. W. Engl, M. Hanke, and A. Neubauer, "Regularization of Inverse Problems." Kluwer Academic Publishers, pp. 127-150, 2000.

[8] C. W. Groetsch, "The Theory of Tikhonov Regularization for Fredholm Equations of the First Kind”. London: Pitman, 1984.

[9] S. Osher, M. Burger, D. Goldfarb, J. Xu, and W. Yin, "An iterative regularization method for total variationbased image restoration," SIAM Multiscale Model. Sim., vol. 4, pp. 460-489, 2005.

[10] M. Elad, "On the origin of the bilateral filter and ways to improve it,’IEEE Trans. Image Process., vol. 11, no. 10, pp. 1141-1151, Oct. 2002.

[11] M. Elad, "Sparse and Redundant Representations: From Theory to Applications in Signal and Image Processing." Berlin: Springer, 2010.

[12] J. Myrheim and H. Rue, "New algorithms for maximum entropy image restoration," CVGIP: Graphical Models and Image Processing, vol. 54, no. 3, pp. 223-238, May 1992.

[13] P. C. Hansen, "Analysis of discrete ill-posed problems by means of the L-curve," SIAM Review, vol. 34, no. 4 , pp. 561-580, December 1992.

[14] A. K.Katsaggelos and S. N. Efstratiadis., "A class of iterative signal restoration algorithms." IEEE Trans. Acoust., Vol. 38, pp.778-786, 1990

[15] L. Landweber, "An iteration formula for Fredholm integral equations of the first kind,"American Journal of Mathematics, vol. 73, no. 3, pp. 615-624, July 1951.

[16] D. G. Luenberger and Y. Ye, "Linear and Nonlinear Programming. Berlin: Springer, 2008

[17] W. H. Richardson, "Bayesian-based iterative method of image restoration," Journal of the Optical Society of America, vol. 62, no1, pp. 55-59, January 1972.

[18] L. B. Lucy, "An iteration technique for the rectification of the obscured distributions," The Astronomical Journal, vol. 79, no. 6, pp. 745-754, June 1974.

[19] H. Stark and P. Oskoui, "High-resolution image recovery from image-plane arrays using convex projections," Journal of the Optical Society of America A, vol. 6, no. 11,pp. 1715-1726, November 1989.

[20] A. M. Tekalp, M. K. Ozkan, and M. I. Sezan, "Highresolution image reconstruction from lower-resolution image sequences and space-varying image restoration," in Proceedings of the IEEE International Conference on Acoustics, Speech, and Signal Processing, vol. 3, March 1992, pp. 169-172 
[21] C.B. Atkins, C. A. Bouman, and J. P. Allebach, "Optimal image scaling using pixel classification," Proceedings of the IEEE International Conference on Image Processing, vol. 3, pp. 864-867, October 2001.

[22] D. Capel and A. Zisserman, "Computer vision applied to super resolution," IEEE Signal Processing Magazine, vol. 20, no. 3, pp. 75-86, May 2003.

[23] Liyakathunisa, and V. K. Ananthashayana, "Super Resolution Blind Reconstruction of Low Resolution Images using Wavelets based Fusion", World Academy of Science, Engineering and Technology 40, pp 177181, 2008.

[24] R. Fergus, B. Singh, A. Hertzmann, S. T. Roweis, and W. T. Freeman, "Removing camera shake from a single photograph," ACM Transactions on Graphics, vol. 25, no. 3, pp. 787-794, July 2006

[25] S. Osher and L. I. Rudin, "Feature-oriented image enhancement using shock filters." SIAM Journal on Numerical Analysis, vol. 27, no. 4, pp. 919-940, August 1990.

[26] J.W. Miskin and D. J. C. MacKay, "Ensemble learning for blind image separation and deconvolution," Advances in Independent Component Analysis. pp.123141, July 2000

[27] Ayers G. R., and Dainty J. C., "Iterative blind deconvolution methods and its applications," Optics Letter, vol. 13, no. 7, July 1988.

[28] Lajendijk, R. L., Biemond J., and Boekee, D. E., "Regularized Iterative Image Restoration with Ringing Reduction. IEEE Transactions on Acoustics, Speech, and Signal Processing, vol. 36, no. 12, 1874-1888, 1988

[29] Kundur D. and Hatzinakos D., "A novel blind deconvolution scheme for image restoration using recursive filtering," IEEE Transactions on Signal Processing, vol. 46, no. 2, pp. 375-390, 1998

[30] I. F. Gorodnitsky and B. D. Rao, "Sparse signal reconstruction from limited data using FOCUSS: A reweighted minimum norm algorithm," IEEE Transactions on Image Processing, vol. 45, no. 3, pp. 600-616, March 1997.

[31] J. M. Bioucas-Dias, M. A. T. Figueiredo, and J. P. Oliveira, "Total variation-based image deconvolution: A majorization-minimization approach," Proceedings of the IEEE International Conference on Acoustics, Speech and Signal Processing, vol. 2, pp. 861-864, May 2006.

[32] J. M. Bioucas-Dias and M. A. T. Figueiredo, "A new twIST: Two-step iterative shrinkage/ thresholding algorithms for image restoration," IEEE Transactions on Image Processing, vol. 16, no. 12, pp. 2992-3004, December 2007

[33] G. Aubert and P. Kornprobst, "Mathematical Problems in Image Processing: Partial Differential Equations and the Calculus of Variations. Berlin: Springer, 2006.

[34] I. Markovsky and S. Van Huffel, "Overview of total least-squares methods," Signal Processing, vol. 87, no. 10, pp. 2283-2302, October 2007.

[35] D. S. G. Pollock, "A Handbook of Time-Series Analysis, Signal Processing and Dynamics. New York: Academic Press, 1999.

[36] W. Dong, L. Zhang, G. Shi and X. Wu, "Image deblurring and supper-resolution by adaptive sparse domain selection and adaptive regularization," in IEEE Trans. on Image Processing, vol. 20, no. 8, pp. 23782386, 2011.

[37] Moacir P. Ponti, Nelson D., A. Mascarenhas, Paulo J., S. G. Ferreira, and Claudio A. T. Suazo, "Threedimensional noisy image restoration using filtered extrapolation and deconvolution,"springer, 2011

[38] Amir Beck and Marc Teboulle, "A Fast Iterative Shrinkage Thresholding Algorithm for Linear Inverse Problems", SIAM Imageing Sciences, Vol. 2, No. 1, pp. 183-202, 2009. 